\title{
OPTIMIZATION OF COUPLING RATIO AND KINEMATICS OF AN UNDERACTUATED ROBOT LEG FOR PASSIVE TERRAIN ADAPTABILITY
}

\author{
Oren Y. Kanner \\ Department of Mechanical Engineering, \\ Yale University, \\ New Haven, Connecticut, USA \\ oren.kanner@yale.edu
}

\author{
Aaron M. Dollar \\ Department of Mechanical Engineering, \\ Yale University, \\ New Haven, Connecticut, USA \\ aaron.dollar@yale.edu
}

\begin{abstract}
This paper investigates how the passive adaptability of an underactuated robot leg to uneven terrain is affected by variations in design parameters. In particular, the ratio between the joint torques, the ratio between the link lengths, and the initial joint rest angles are varied to determine configurations that allow for maximum terrain roughness adaptability while minimizing the transmission of disturbance forces to the body. The results show that a proximal/distal joint torque coupling ratio of 1.58 , proximal/distal leg length ratio of 0.5 , and an initial proximal joint angle of -49 degrees maximize the terrain variability over which the robot can remain stable by exerting a near-constant vertical reaction force while minimizing lateral force and moment disturbances. In addition, the spring stiffness ratio allows for a tradeoff to be made between the different performance metrics.
\end{abstract}

\section{INTRODUCTION}

Good performance over uneven and rough terrain is a key factor for the successful implementation of many legged robotic systems. Besides bipeds, work in this area has focused on legged systems that fall into two main groups: 1) simpler systems intended to run mostly open-loop, many of which utilize compliance in the legs and/or body (e.g. [1-4]), and 2) more complex systems that close the loop around a variety of sensors in order to control leg placement, stiffness, or force (e.g. [5-7]). While there are clear advantages and shortcomings to both approaches, it is desirable to develop systems that have a good balance of performance, reliability, and cost.

In this paper, we begin to investigate the development of legged robots that utilize adaptive underactuation, through

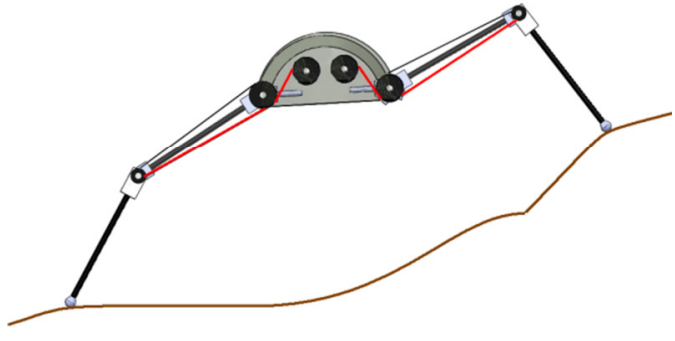

Figure 1. Diagram of a tendon-driven underactuated adaptive robot standing on uneven terrain. Due to the underactuated leg mechanism the robot will be stable across a wide range of ground heights.

differential transmission designs, to allow the legs to naturally adapt to rough terrain to an extent that mechanical compliance alone typically cannot enable. In other words, an underactuated leg would allow a legged robot to find stable footing on unknown, uneven terrain without the need for closed-loop control and active sensing of the terrain. These types of mechanisms have been used extensively in robotic hands in order to enable passive adaptation to a wide range of object sizes, shapes, and positions/orientations in a purely open-loop manner (e.g. [8-10]). However, as far as the authors are aware, they have yet to be applied to legged robotic systems.

In this initial study, we examine how variations in the mechanical parameters of a two-link leg with rotational joints affect its performance in unstructured, rough terrain. In particular, we investigate how changes in the initial joint angles, the ratio between the link lengths, and the ratio between the joint torques affect the ability of the leg to exert a nearconstant vertical force on the ground (equal to a $1 / n$ portion of robot body weight for a n-legged robot), with minimal horizontal force and moment disturbances. These are studied across a wide range of variations in ground height in order to 


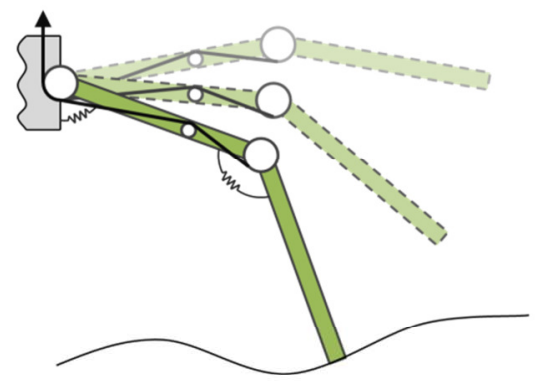

Figure 2. Diagram of a 2-DOF underactuated leg showing its actuation motion. Note the single tendon driving both joints; it is rigidly attached to the distal joint pulley and couples both joints with a fixed torque ratio.

find the leg configurations that adapt to the widest range of terrain roughness without actively sensing and modulating the actuator force on the leg - effectively providing the best openloop passive terrain adaptability while adding minimal disturbance forces to the body of the robot.

We begin this paper by presenting our underactuated leg model and description of terms. We then describe our simulation, including the model parameters that are most relevant and performance metrics used to evaluate variations in those, and then show how performance with respect to those metrics varies as the model parameters vary. Finally, we discuss the implications from these results as they apply to the design of practical passively adaptive underactuated legged robotic systems.

\section{NOMENCLATURE}

$\begin{array}{ll}T & \text { Tendon tension } \\ r_{i} & \text { Joint pulley radius } \\ \theta_{i} & \text { Joint angle } \\ k_{i} & \text { Joint spring constant } \\ \theta_{i, 0} & \text { Joint initial angle } \\ \ell_{i} & \text { Leg segment length } \\ F_{x}, F_{y} & \text { Ground reaction forces }\end{array}$

\section{UNDERACTUATED LEG MODEL}

\section{Basic Leg Description}

Fig. 1 shows a cross-section of an adaptive mobile robot standing on uneven terrain. Each of its 2-DOF legs is actuated through a single tendon, which allows the leg to reach a wide range of ground heights. Due to the coupling between the two joints and the natural compliance of the leg, the robot can exert different forces on the ground, potentially allowing it to remain stable despite changes in the height of the ground. For the purposes of this paper we will only consider a single robot leg.

The use of revolute joints as opposed to prismatic joints was not arbitrary. Revolute joints are easily driven and well understood, and also allow for a larger set of achievable leg trajectories. While prismatic legs might achieve good force performance, their trajectories would have to be largely

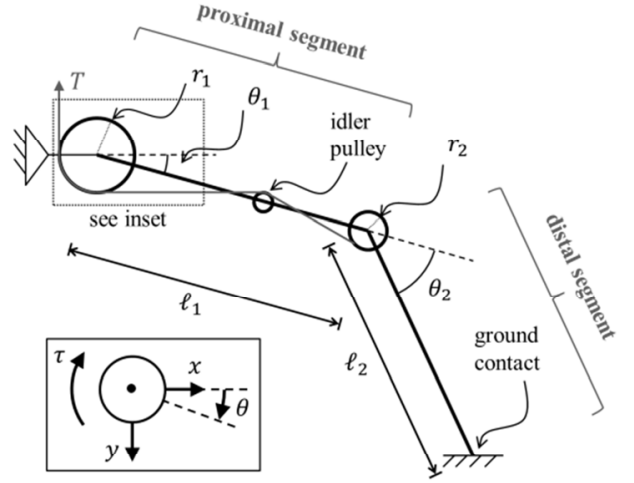

Figure 3. Representative 2-DOF underactuated robot leg with relevant parameters and components labeled. Note the inset coordinate frame illustration and definition of $\theta_{2}$ relative to the proximal link.

vertical, requiring the body frame to be wide to provide a stable stance. Revolute legs can provide a more sprawled posture while still providing the required forces for stable weight support.

Fig. 2 shows the actuation motion of a generic two-link underactuated robot leg as described above. Each joint incorporates a spring to return the leg to some default position in the absence of tendon tension, with the actuation of the leg being used to lower it to the ground and support the robot's weight. The tendon is rigidly attached to the pulley at the distal joint, which, in turn, is rigidly attached to the distal link. Both the idler pulley on the proximal link as well as the pulley at the proximal joint are free-spinning, and we assume frictionless bearings throughout the leg. We also assume that the leg is mass-less, and therefore ignore inertial effects throughout this analysis.

We define the torque at each joint as the tendon tension multiplied by the radius of the pulley at the joint, or $\tau_{i}=\operatorname{Tr}_{i}$, where $\tau_{i}$ is the torque in joint $i, T$ is the tendon tension, and $r_{i}$ is the effective pulley radius of joint $i$. When the leg is unconstrained, e.g. not in contact with the ground, an increase in the joint torque corresponds to an increase in the joint angle, or $\Delta \theta_{i}=\tau_{i} / k_{i}$, where $\Delta \theta_{i}=\theta_{i}-\theta_{i, 0}$ is the difference between the current joint angle and the initial joint angle and $k_{i}$ is the spring constant of joint $i$. The initial joint angles $\theta_{i, 0}$ defines the initial configuration of the leg. Fig. 3 shows a complete leg with relevant parameters and components labeled and the general coordinate frame used in the model; the origin is at the proximal joint of the leg with positive angles denoting a leg position below the $x$ axis and positive torques following the angle coordinate convention.

\section{Reaction Forces}

In order to evaluate the performance of a given leg design we need to know the reaction forces at the foot of the leg for a given leg configuration and ground height. The following model assumes that the robot body is fixed in space, and calculates the reaction force required to ensure that the leg is in 
static equilibrium. As we will discuss later, it is useful to decompose the ground reaction forces into vertical and horizontal components (with respect to our global coordinate frame). Thus it should be kept in mind that our end goal is the evaluation of the two reaction force components, $F_{x}$ and $F_{y}$.

We begin with an underactuated leg in contact with the ground in some configuration. We use the ground height and the leg parameters to determine that configuration, incrementing the tension in the leg until the foot reaches the ground. The position of the foot can easily be evaluated as:

$$
\begin{aligned}
& x=\ell_{1} \cos \left(\theta_{1}\right)+\ell_{2} \cos \left(\theta_{1}+\theta_{2}\right) \\
& y=\ell_{1} \sin \left(\theta_{1}\right)+\ell_{2} \sin \left(\theta_{1}+\theta_{2}\right)
\end{aligned}
$$

Once we have determined the contact configuration of the leg, we use static equilibrium to determine the ground reaction forces generated by the leg. The tendon tension is set to a fixed amount, and we use the sum of moments about each joint to solve for the reaction force. Looking at the distal segment, we can express that sum of moments as:

$$
\tau_{2 a}-\ell_{2}\left[F_{x} \sin \left(\theta_{1}+\theta_{2}\right)+F_{y} \cos \left(\theta_{1}+\theta_{2}\right)\right]=0
$$

where $\tau_{2 a}=T r_{2}-k_{2} \Delta \theta_{2}$ is the net torque at the distal joint. Treating the entire leg as a rigid object and the distal joint torque as purely internal, we have the sum of moments at the proximal joints as:

$$
\tau_{1 a}-F_{x} \cdot y-F_{y} \cdot x=0
$$

where $\tau_{1 a}=\operatorname{Tr}_{1}-k_{1} \Delta \theta_{1}$ is the net torque at the proximal joint. We now have two linear equations with two unknowns that we can solve for. Solving (3) and (4) for $F_{x}$ and $F_{y}$ we get:

$$
\begin{aligned}
F_{x} & =\frac{\tau_{2 a} \cdot x-\tau_{1 a} \ell_{2} \cos \left(\theta_{1}+\theta_{2}\right)}{\ell_{2} \sin \left(\theta_{1}+\theta_{2}\right) \cdot x-\ell_{2} \cos \left(\theta_{1}+\theta_{2}\right) \cdot y} \\
F_{y} & =\frac{\tau_{1 a} \ell_{2} \sin \left(\theta_{1}+\theta_{2}\right)-\tau_{2 a} \cdot y}{\ell_{2} \sin \left(\theta_{1}+\theta_{2}\right) \cdot x-\ell_{2} \cos \left(\theta_{1}+\theta_{2}\right) \cdot y}
\end{aligned}
$$

Equations (1) and (2) allow us to generate the unconstrained motion of the leg, e.g. the progression of joint angles as it lowers to the ground. In addition, Eqns. (5) and (6) allow us to calculate the ground reaction forces for a leg given its joint angles and final tendon tension. For a given set of leg design parameters (to be discussed in the following section), Eqns. (1) and (2) were used to calculate the motion of the leg as the tendon tension was increased. The leg configuration was recorded at a series of equally spaced ground heights until the leg reached its maximum vertical displacement; this was considered to be the end of the useful trajectory of the leg. For each recorded configuration, Eqns. (5) and (6) were then used to evaluate the reaction forces. This data was then analyzed in the context of the performance metrics described in the following section.

\section{ANALYSIS AND RESULTS}

\section{Normalized Parameters / Assumptions}

To simplify our analysis the definition of an underactuated leg was broken down into two non-dimensional parameters and a single intial joint angle term. The first non-dimensional parameter was the segment length ratio, defined as $R_{L}=\ell_{1} / \ell_{2}$. The second parameter was the joint torque coupling ratio, defined as $R_{T}=\tau_{1} / \tau_{2}$. The initial proximal joint angle, $\theta_{1,0}$, was used as the final design parameter. In order to eliminate the initial distal joint angle as an independent parameter the rest configuration of the leg was constrained such that the foot always started at $y=0$, the horizontal plane of the robot body. This required that $\theta_{2,0}=-\sin ^{-1}\left(R_{L} \sin \left(\theta_{1,0}\right)\right)-\theta_{1,0}$.

The actual dimensions of the leg were normalized for simplicity. First, each segment length was normalized by some nominal length and the total leg length was constrained to a normalized value of 2 (such that with $R_{L}=1$ we would have $L_{1}=L_{2}=1$ ). The joint pulley radii were similarly normalized by the nominal segment length, as were the joint spring constants. In addition, all the forces were normalized by the final tendon tension to remove its effects from the results.

Rather than include the spring constants as separate design parameters, they were assumed to be equal (e.g. $R_{k}=k_{1} / k_{2}=$ 1 ) and set to a fairly small value. While the spring constants determine the ratio between proximal and distal joint angles, nominally, they only exist to return the leg to its initial configuration, and therefore their effect on the actuation of the leg was expected to be negligible. That being said, we will return to the spring constant ratio later in our discussion. One important assumption made in the analysis was that the proximal joint of the leg was pinned in space (i.e. the robot body would not move relative to the ground) and that the leg would not re-configure once it made contact with the ground. In the absence of a largely horizontal tangential reaction force, one that would overcome static friction, the latter assumption should always be valid as there is no reason for the leg to reconfigure.

One final point to note is that the parameters evaluated only within physically-realistic bounds. The lower and upper bounds of the kinematic parameters (e.g. pulley radii and leg lengths) were chosen such that a robot could reasonably be constructed with those specifications (e.g. no infinitesimal pulley radii), and any designs with interfering parts (such as overlapping pulleys) were ignored.

\section{Performance Metrics}

The performance of an underactuated leg in this analysis was determined by three metrics. The first metric was the 

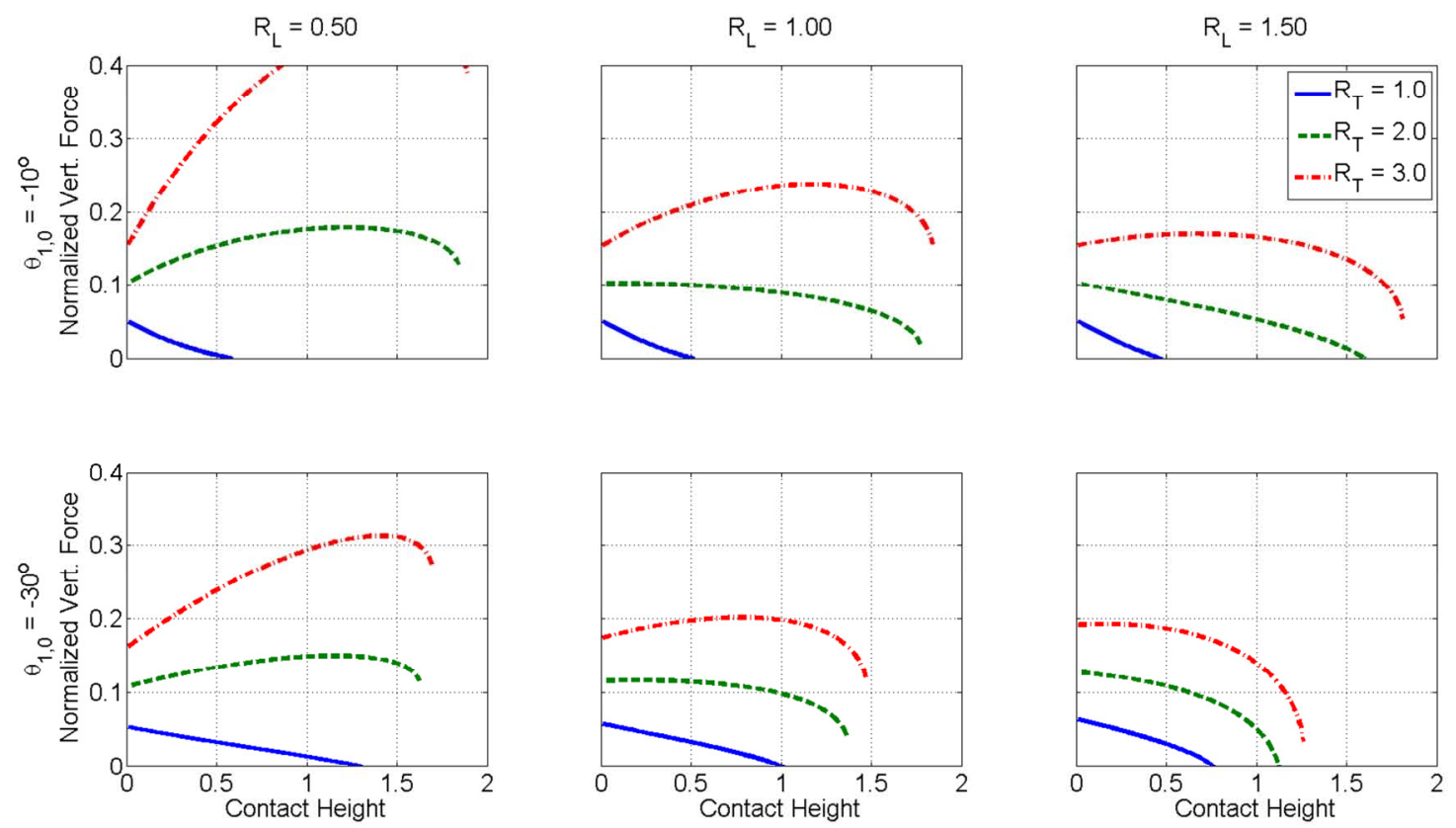

Figure 4. Comparison of vertical reaction force vs. ground contact height profiles for a number of different leg configurations / designs. Note the change in the overall length of the profiles as well as in their shapes (longer / flatter is better). Each column differs in terms of $R_{L}$ (link length ratio) while each row differs in terms of $\theta_{1,0}$ (initial joint angle). For each subplot, $R_{T}$ (joint torque coupling ratio) increases from 1 to 3 as the curves go from bottom to top.

effective range of the leg, or the lowest ground height the leg was capable of reaching $\left(y_{\max }\right)$. This is important since the total length of the leg was fixed across all of the simulations, so it can be viewed as the portion of the leg's length that can be used to reach the ground.

The next two metrics were related to the ground reaction forces exerted by the leg. As described previously, the reaction force was decomposed into its vertical $\left(F_{y}\right)$ and horizontal $\left(F_{x}\right)$ components under the assumption that the global coordinate frame was aligned with positive $y$ in the direction of gravity. In this case, $F_{y}$ would be supplied by the robot's weight (or some fraction depending on the number of legs) while $F_{x}$ would be supplied by the frictional force between the foot and the ground.

In our model, the reaction forces are determined to be the set of forces that will ensure that the robot leg is in static equilibrium (assuming that the body is fixed). If the reaction forces do not match the model, the leg would not be in equilibrium and therefore might be unable to support the robot. The only mechanism through which $F_{y}$ can be generated is the weight of the robot, and this force is constant regardless of leg configuration. Therefore, we desire a leg for which $F_{y}$ is effectively independent of ground height. Similarly, the only mechanism through which $F_{x}$ can be generated is the friction between the foot and the ground. Since this is constrained by the constant normal force, we desire a leg for which minimal horizontal reaction forces are necessary for static equilibrium.

In order to convert those design requirements into numerical quantities, we evaluated each leg design at a number of evenly spaced ground heights and generated force profiles for each. The $F_{y}$ profile for an ideal leg would simply be constant. Note that the magnitude of $F_{y}$ is not very important as the tendon tension value for a given robot design can be set in order to generate the appropriate reaction forces to support a given robot weight. The coefficient of variation $(\mathrm{CV})$ was used to evaluate how "constant" the force was over the range of ground heights. The $C V$ is a normalized measure of the spread of a data set, and is evaluated by dividing the standard deviation of the set by the absolute value of its mean, i.e. $C V(a)=\sigma(a) /|\mu(a)|$. The $F_{x}$ profile for an ideal leg would be close to zero over the entire range of ground heights, or, more precisely, would be small relative to $F_{y}$ such that it could be supported by friction. This was evaluated using the average of the ratio between the absolute values of the two forces, i.e. $\operatorname{avg}\left(\left|F_{x}\right| /\left|F_{y}\right|\right)$. For both of our force-related performance metrics, lower values mean better performance.

\section{Simulation Results}

Over 500,000 different leg designs were evaluated using the previously defined model. $R_{L}$ was varied from 0.25 to 1.25 , $R_{T}$ was varied from 0.25 to 4 , and $\theta_{1,0}$ was varied from $-70^{\circ}$ to $-1^{0}$. A small sample of the force profiles generated by the simulation can be seen in Figs. 4 and 5. The first thing to notice is that the effective range of the legs $\left(y_{\max }\right)$ varies significantly between different designs. In addition, there are a number of $F_{y}$ profiles that, even just by inspection, appear to be fairly constant, while there are also a number of profiles that are far from constant. Similarly, there are some $F_{x}$ profiles with 

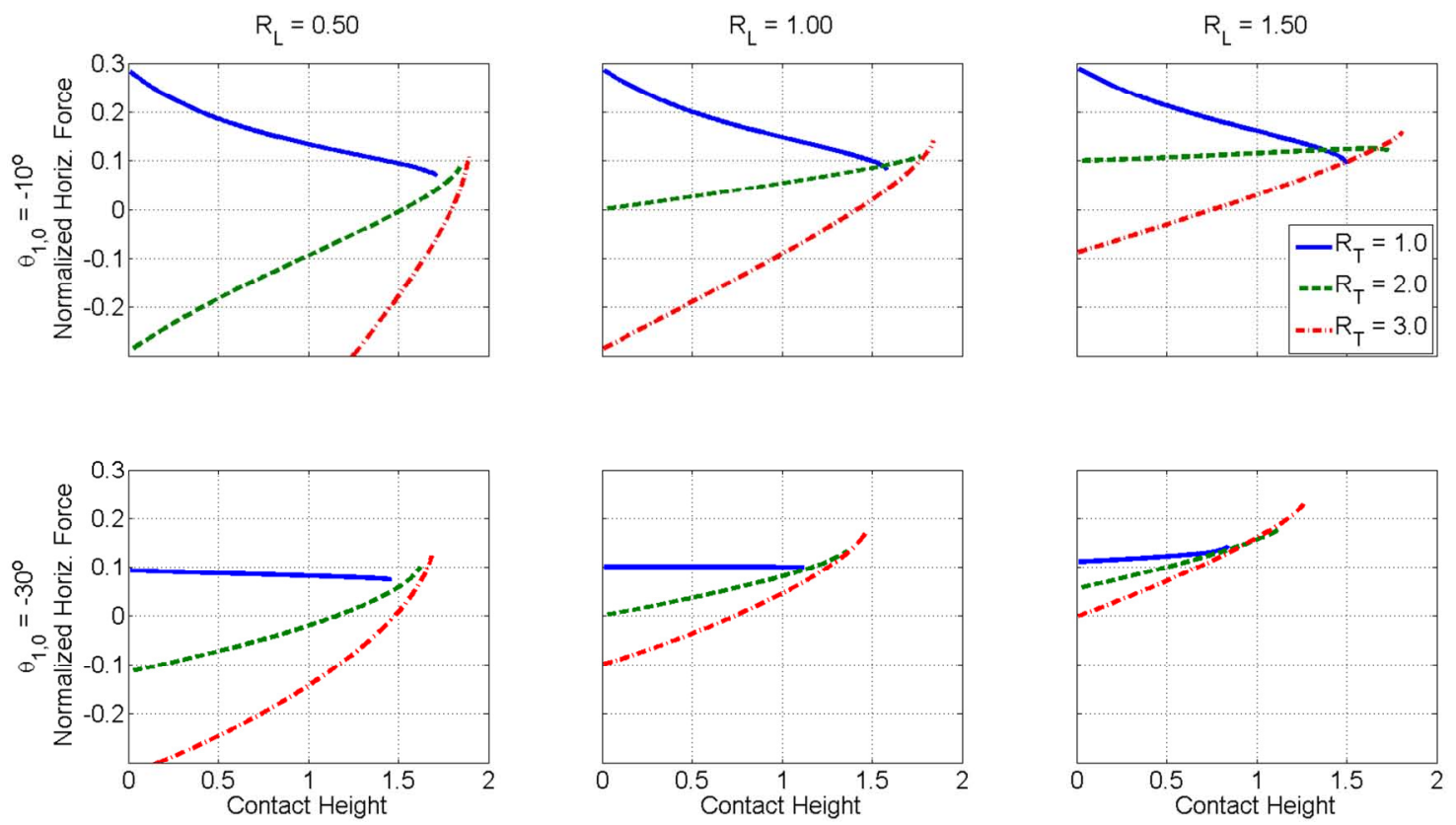

Figure 5. Comparison of horizontal reaction force vs. ground contact height profiles for a number of different leg configurations / designs. Note the change in the overall length of the profiles as well as in their shapes (longer / centered on zero is better). Each column differs in terms of $R_{L}$ (link length ratio) while each row differs in terms of $\theta_{1,0}$ (initial joint angle). For each subplot, $R_{T}$ (joint torque coupling ratio) increases from 1 to 3 as the curves go from top to bottom.

relatively low magnitudes across the entire range of ground heights, while there are also a number of profiles with extremely high magnitudes. It is clear that a more rigorous investigation into the effects of the design parameters on the performance metrics will be necessary to make an informed design decision.

\section{DISCUSSION}

\section{Performance Study}

Fig. 6 shows contours of all three performance metrics (one per column), as a function of $\theta_{1,0}$ (vertical axis) and $R_{T}$ (horizontal axis), for several values of $R_{L}$ (rows). First looking at $y_{\min }$, as previously discussed, an optimal leg would reach as far as possible, allowing for the greatest variability in ground height. In Figs. 4 and 5 we noted some general trends regarding the effect of the three design parameters on $y_{\max }$, and we will now investigate them more formally. The first column of Fig. 6 shows a series of contour plots of $y_{\max }$ for fixed values of $R_{L}$, exposing the effect $R_{T}$ and $\theta_{1,0}$ have on the metric. We notice that smaller / less extreme values of $\theta_{1,0}$ result in better performance. This makes sense because the straighter the leg starts out, the longer it can be actuated before the distal segment starts to swing up (and therefore the lower it can reach). We also notice that the smaller $R_{L}$ is, the better our performance as well, but with a weaker effect. This makes sense because in that case (small $R_{L}$ ) the length of the proximal segment gets smaller and smaller and therefore the leg behaves like a single segment whose maximum reach is simply the increasing length of the distal segment. Finally, we notice that larger values of $R_{T}$ result in better performance as well. The poor performance of legs with low $R_{T}$ makes sense because in those cases the distal joint moves much more than the proximal joint, so the proximal segment travels very little before the distal segment starts swinging back up.

We will now consider the two force-related metrics. The second column of Fig. 6 shows a series of contour plots of $C V\left(F_{y}\right)$ for fixed values of $R_{L}$. We see that there is an optimal region with respect to $C V\left(F_{y}\right)$ that moves as $R_{L}$ increases. For smaller values of $R_{L}$ this region occupies moderate values of $\theta_{1,0}$ and small values of $R_{T}$. As $R_{L}$ increases we see a dramatic decrease in performance across the entire resulting parameter space with decent performance only occurring at extreme values of $\theta_{1,0}$, which is suboptimal for $y_{\max }$. While the contours seem to indicate an optimum for $C V\left(F_{y}\right)$ at some more extreme value of $\theta_{1,0}$, angles less than $-70^{\circ}$ result in extremely short reach to the point that the leg is almost ineffective.

We see a slightly different pattern in the second forcerelated performance metric, or $\operatorname{avg}\left(\left|F_{x}\right| /\left|F_{y}\right|\right)$. The third column of Fig. 6 shows a series of contour plots of $\operatorname{avg}\left(\left|F_{x}\right| /\right.$ $\left.\left|F_{y}\right|\right)$ for fixed values of $R_{L}$. We see that there is an optimal performance region for each value of $R_{L}$ and that the magnitude of the metric tends to increase slightly as $R_{L}$ increases. The location of the optimal performance region changes as $R_{L}$ changes, but as $R_{L}$ increases the optimal value of $R_{T}$ changes more significantly to the point where at $R_{L}=1$ the optimal $R_{T}$ is beyond the scope of our parameter space. In general, more 

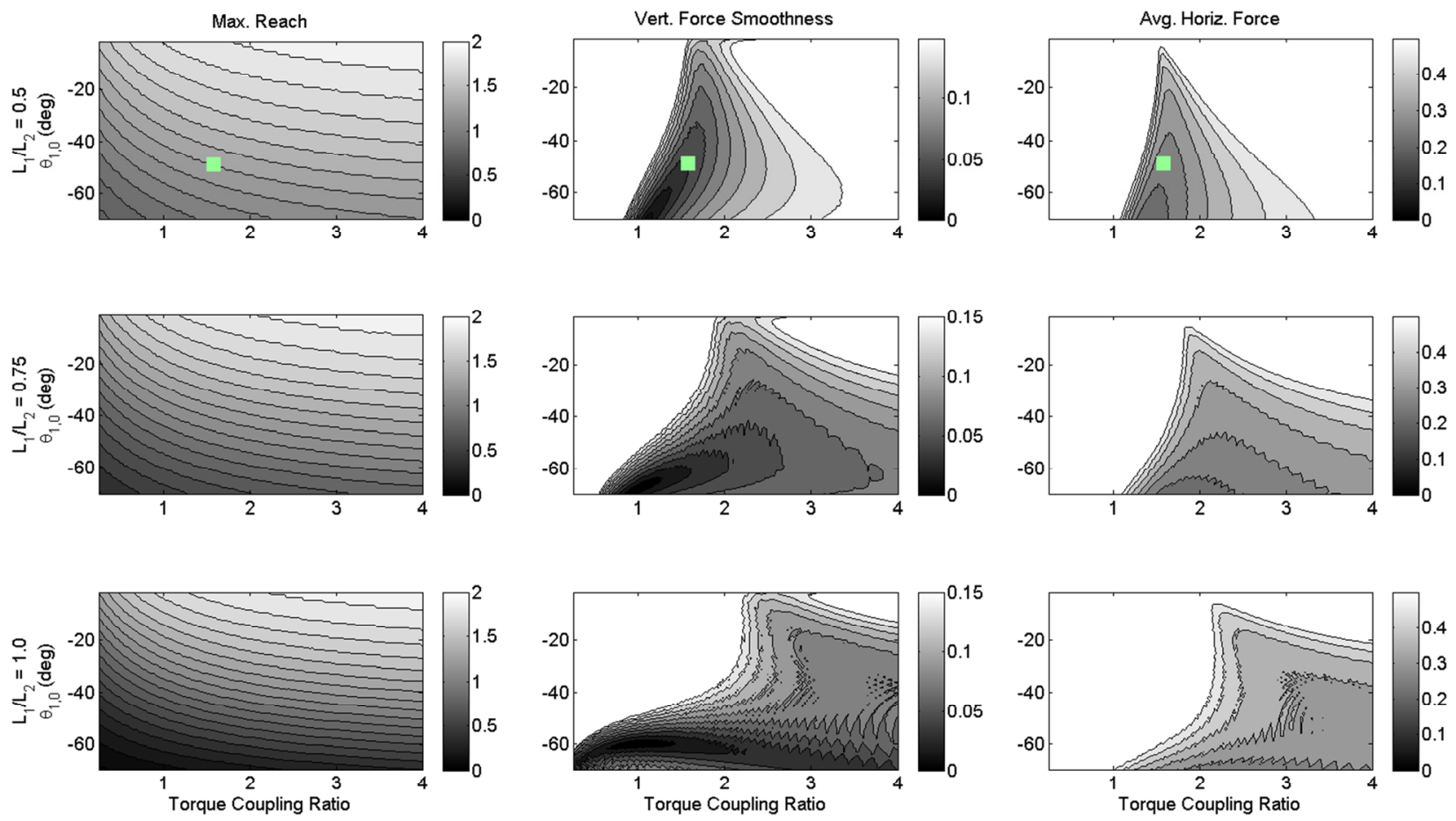

Figure 6. Contour plots of the three performance metrics, $y_{\max }$ (maximal leg travel), $C V\left(F_{y}\right)$ (smoothness of vertical force profile), and $a v g\left(\left|F_{x}\right| /\left|F_{y}\right|\right)$ (average lateral force) for three separate values of $R_{L}, 0.5,0.75$, and 1.0 (top to bottom). For the first column, lighter is preferable; for the second two, darker is preferable. Note the change in behavior as $R_{L}$ increases. The marker on the top row of subplots represents an optimal configuration that balances all three metrics.
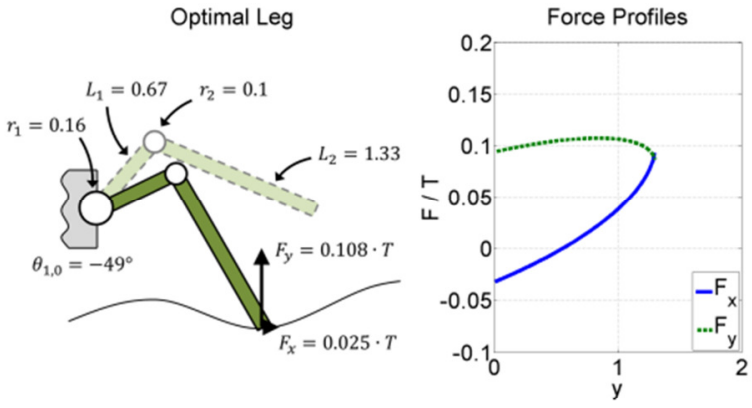

Figure 7. Diagram of optimal leg design for $R_{k}=1$ with reaction force profiles normalized to the tendon tension. On the left, note the normalized dimensions of the leg and the reaction force vectors. On the right, note that $F_{y}$ is relatively smooth across the entire range of ground heights, and is almost always substantially larger than $F_{x}$.

extreme values of $\theta_{1,0}$ give better performance with respect to this metric, but the difference over the range of $\theta_{1,0}$ at the optimal value of $R_{T}$ is fairly small.

It is clear that $R_{L}=0.5$ gives the best general performance out of the three values displayed in Fig. 6. For each of the subplots for that value of $R_{L}$, there is a marker representing the optimal configuration that balances all three metrics. The optimal value of $\theta_{1,0}$ is somewhat extreme, resulting in somewhat sub-optimal $y_{\max }$ performance, but otherwise the optimal leg chosen does well overall. Fig. 7 shows the optimal design at an arbitrary point along its trajectory as well as its force profiles. We see that the leg has decent reach (around

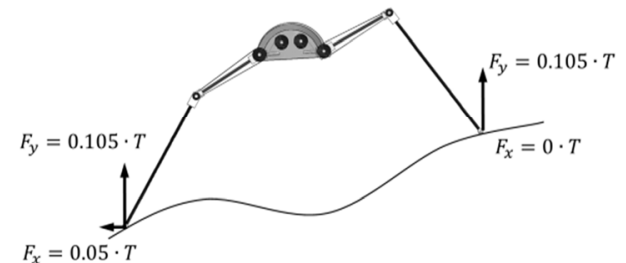

Figure 8. Diagram of adaptive legged robot standing on rough terrain with approximate reaction force vectors (derived from optimal configuration) drawn and labeled. The ground heights are 0.5 on the right and 1.1 on the left.

1.3), has a fairly constant $F_{y}$ profile, and that $F_{y}$ is generally much larger than $F_{x}$. Fig. 8 shows the representative adaptive legged robot from Fig. 1 (modified to match our optimal configuration) with approximate reaction force vectors based our model. We see that the vertical component of both reaction force vectors is basically constant, as expected, and also that both vectors are primarily vertical, indicating that relatively small frictional forces would be required for static equilibrium. It is reasonable to conclude that this robot would be stable despite the difference in ground height on either side. Rather than conclude our analysis here, we will briefly investigate the effect of the spring constant ratio for completeness.

As mentioned before, the springs serve to return the leg to its default position in the absence of tendon tension. However, the ratio of spring stiffness between the two joints, combined 

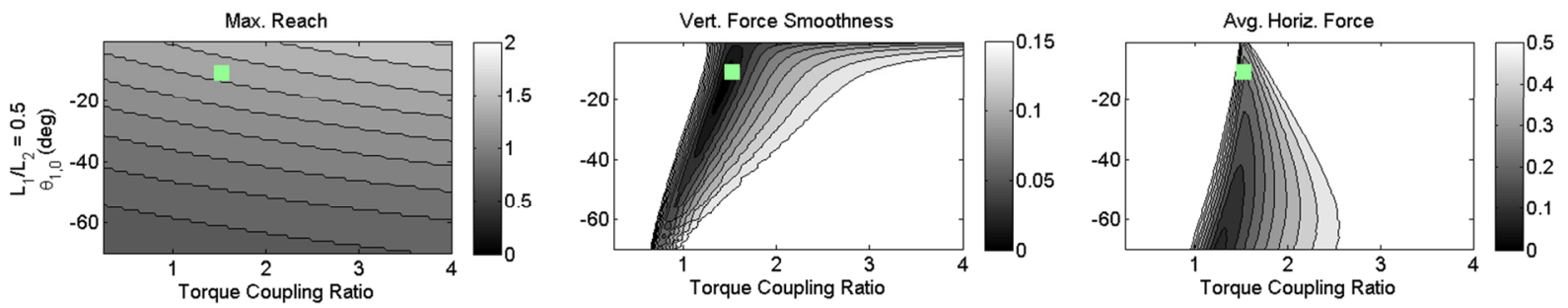

Figure 9. Contour plots of the three performance metrics, $y_{\max }$ (maximal leg travel), $C V\left(F_{y}\right)$ (smoothness of vertical force profile), and avg $\left(\left|F_{x}\right| /\left|F_{y}\right|\right)$ (average lateral force) for $R_{k}=10\left(R_{L}=0.5\right)$. For the first subplot, lighter is preferable; for the second two, darker is preferable. The marker on each subplot represents an optimal configuration that balances all three metrics. Note the performance difference as compared to the $R_{k}=1$ designs, especially with regards to the optimum performance location.

with the torque ratio, also determines the relative motion between the two joints. To put that in mathematical terms, $R_{\theta}=\Delta \theta_{1} / \Delta \theta_{2}=R_{T} / R_{k}$. This is important in determining the configuration of the leg (e.g. the joint angles) when it makes contact at a given ground height, but is otherwise irrelevant. To see if this would impact the performance of the leg, we ran a series of simulations with $R_{k}=10$, an order of magnitude larger than the results we showed previously, and checked the same three metrics.

Fig. 9 shows a contour plot for each of our three performance metrics for $R_{L}=0.5$ and $R_{k}=10$. We see similar behavior with regard to $\operatorname{avg}\left(\left|F_{x}\right| /\left|F_{y}\right|\right)$ and to a lesser extent with $y_{\max }$, but the behavior of $C V\left(F_{y}\right)$ is quite different. The optimal region shifts significantly towards less extreme values of $\theta_{1,0}$, with the optimal range of $R_{T}$ shifting slightly higher. In addition, if we look at the optimal configuration that balances all three metrics, we see that the performance metric values for that configuration are similar to those for the optimal $R_{k}=1$ leg, and also that the location of that configuration within the various optimal regions (especially for the force metrics) is similar as well. That being said, since the higher spring constant lessens the motion of the proximal joint, we see similar, if not worse, performance with respect to $y_{\max }$.

In order to investigate the effect of $R_{k}$ we will compare the two optimal configurations and their performance. Table 1 shows both the design parameters for each optimal configuration as well as the values of all three performance metrics for both legs. We see that their performance is fairly comparable, with a small increase in the force performance of the $R_{k}=10$ leg coming at the expense of its efficiency, e.g. the ground reaction forces are roughly $20 \%$ lower for the $R_{k}=10$ leg with the same tendon tension. Therefore, it seems as if varying $R_{k}$ allows us to make trade-offs with respect to different performance characteristics, depending on our priorities.

\section{Limitations and Future Work}

There are a number of limitations to this work and a number of things that will be added as this project progresses. One major limitation is that this model only deals with the issue
Table 1: Optimal Underactuated Leg Designs

\begin{tabular}{ccc}
\hline \hline Variable / Metric & $R_{k}=1$ & $R_{k}=10$ \\
\hline$R_{L}$ & 0.5 & 0.5 \\
$R_{T}$ & 1.575 & 1.525 \\
$\theta_{1,0}$ & $-49^{\circ}$ & $-11^{\circ}$ \\
\hline$y_{\max }$ & -1.3 & -1.33 \\
$C V\left(F_{y}\right)$ & 0.043 & 0.015 \\
$\operatorname{avg}\left(\frac{\left|F_{x}\right|}{\left|F_{y}\right|}\right)$ & 0.27 & 0.26 \\
\hline \hline
\end{tabular}

of weight support, ignoring any issues related to actual locomotion (i.e. propulsive force). In addition, the model assumes level ground, whereas slanted ground would change the relationship between the required vertical force for weight support and the friction force necessary to prevent the foot from slipping on the ground. It would also be greatly strengthened through the inclusion of the mechanism's "equilibrium point" $[9,11]$ to evaluate potentially unstable configurations. That being said, this work does provide insight into the force performance of underactuated 2-DOF robot legs as it relates to the different design parameters.

Looking forward, in addition to addressing the limitations above, we will be working towards the development of a physical prototype. In this, we will be implementing betweenleg coupling, e.g. actuating multiple legs with a single tendon using a differential transmission, which could vastly improve the stability of such an adaptive robot as well as reduce the required number of actuators. The nature of the specific implementation will be based on a similar, in-depth design study to find coupling configurations that give the best performance.

\section{REFERENCES}

[1] P. Birkmeyer, K. Peterson, and R. S. Fearing, "Dash: A dynamic $16 \mathrm{~g}$ hexapedal robot," Proceedings of the 2009 IEEE/RSJ International Conference on Intelligent Robots and Systems, pp. 2683-2689, 2009.

[2] K. A. Daltorio, T. E.Wei, A. D. Horchler, L. Southard, G. D.Wile, R. D. Quinn, S. N. Gorb, and R. E. Ritzmann, "Mini-Whegs TM Climbs Steep Surfaces Using Insect-inspired Attachment Mechanisms," International Journal of Robotics Research, v. 28, n.2, pp. 285-302, 2009. 
[3] S. Kim, J. E. Clark, and M. R. Cutkosky, "iSprawl: Design and Tuning for High-speed Autonomous Open-loop Running," International Journal of Robotics Research, v. 25, n. 9, pp. 903-912, 2006.

[4] U. Saranli, M. Buehler, and D. E. Koditschek, "RHex: A simple and highly mobile hexapod robot," International Journal of Robotics Research, v. 20, n. 7, pp. 616-631, July 2001.

[5] B. Klaassen, R. Linnemann, D. Spenneberg, and F. Kirchner, "Biomimetic walking robot scorpion: Control and modeling," J. Robotics and Autonomous Systems, v. 41, n. 2-3, pp. 69 - 76, 2002.

[6] B. McBride, R. Longoria, and E. Krotkov, "Off-road mobility of small robotic ground vehicles: Measuring the performance and intelligence of systems," Proceedings of the Workshop on Performance Metrics for Intelligent Systems NIST Special Publication 1014, pp. 405-412, 2003.

[7] M. Raibert, K. Blankespoor, G. Nelson, R. Playter, and T. B. Team, "Bigdog, the Rough-Terrain Quadruped Robot," In Proceedings of the $17^{\text {th }}$ World Congress of the International Federation of Automatic Control, pp. 10822-10825, 2008.

[8] S. Hirose and Y. Umetani, "The development of soft gripper for the versatile robot hand," Mechanism and Machine Theory, v. 13, pp. 351359,1978 .

[9] L. Birglen, C. Gosselin, and T. Laliberté, Underactuated Robotic Hands, Springer, 2008.

[10] A.M. Dollar and R. D. Howe, "The Highly Adaptive SDM Hand: Design and Performance Evaluation," International Journal of Robotics Research, v. 29, n. 5 , pp. $585-597,2010$.

[11] R. Balasubramanian, J.T. Belter, and A.M. Dollar, "External Disturbances and Coupling Mechanisms in Underactuated Hands," Proceedings of the 2010 ASME International Design Engineering Technical Conferences, Mechanisms and Robotics Conference (IDETC 2010), pp. 175-184, 2010. 